\title{
Evolución clínica y radiológica en el tratamiento de hernia discal con prótesis PDN-SOLO con ancla: seguimiento mínimo a tres años
}

\author{
Clinical and radiological development in the treatment of \\ intervertebral disc with prosthesis PDN-SOLO with anchors: follow-up \\ to a minimum of three years \\ Evolução clínica e radiológica no tratamento da hérnia discal com \\ prótese PDN-SOLO com ancora: seguimento mínimo por três anos
}

\author{
Alejandro Antonio Reyes-Sánchez' \\ Nicasio Arriada ${ }^{2}$ \\ Víctor Miramontes $\mathrm{M}^{3}$ \\ Armando Alpizar A. ${ }^{3}$ \\ Luis M. Rosales $\mathrm{O}^{4}$
}

\section{RESUMEN}

Objetivo: las prótesis de núcleo (PDN$S O L O)$ reconstruyen la anatomía y preservan la biomecánica del disco intervertebral con un índice de migración de $10 \%$ y de hundimiento de 19\%. Por eso, proponemos un sistema de fijación para evitar estas complicaciones. Métodos: fueron utilizados veinte pacientes con seguimiento mínimo de tres años. La valoración de dolor con EVA, función con Escala de Oswestry (EO) y disco por estudios radiográficos y resonancia magnética fueron aplicadas. El análisis estadístico fue por $t$ de Student. Resultados: El grupo comprende 8 mujeres $y$ 12 hombres con una edad promedio de 37.6 años. Los valores preoperatorios de EVA fueron 7.6 puntos y EO de 67\%, la altura del espacio intervertebral (El) de $7.67 \mathrm{~mm}$. A tres años, EVA de dolor de 0.7 y EO a menos de 6\% $(p<0.000)$. Incremento altura disco promedio $2 \mathrm{~mm}$.

\section{RESUMO}

Objetivo: as próteses de núcleo (PDNSOLO) reconstituem a anatomia e preservam a biomecânica do disco intervertebral, com um índice de migração de $10 \%$ e de afundamento de $19 \%$. Por isso, foi proposto um sistema de fixação para evitar essas complicações. Métodos: foram utilizados 20 pacientes com seguimento mínimo de três anos. A avaliação da dor com EVA, função com Escala de Oswestry (EO) e disco por estudos radiológicos e ressonância magnética foram aplicados. A análise estatística foi por teste $t$ de Student. Resultados: Fizeram parte do estudo 12 homens e $8 \mathrm{mu}-$ lheres, com média de idade de 37,6 anos. Valores pré-operatórios da EVA foram de 7,6 pontos e EO de $67 \%$, a altura do espaço intervertebral (EI) de 7,67 mm. Durante três anos, a EVA de dor foi de 0,7 e a EO foi menos de $6 \%(p<0,000)$. Houve aumento da altura discal média

\section{ABSTRACT}

Objective: The prosthetic nucleus (PDN-SOLO) reconstruct the anatomy and preserve the biomechanics of intervertebral disc with a migration rate of $10 \%$ and sinking rate of $19 \%$. Therefore, a fixing system to avoid these complications was proposed. Methods: A total of 20 patients were followed-up for at least three years. The assessment of pain with VAS, Oswestry Scale function (OS) and disk by radiological studies and magnetic resonance imaging were applied. Statistical analysis was made by Student's t-test. Results: The subjects were 12 men and 8 women with a mean age of 37.6 years. VAS preoperative values were 7.6 points and OS 67\%, the height of intervertebral space was $7.67 \mathrm{~mm}$. For three years, the VAS pain score was 0.7 and OS less than $6 \%(p<0.000)$. There was an increase of average disc height

\footnotetext{
Trabajo realizado en el Instituto Nacional de Rehabilitación - INR - México.

'Jefe de División de Cirugía Especial del Instituto Nacional de Rehabilitación - INR - México.

${ }^{2}$ Residente, especialidad de Cirugía de Columna Vertebral en el Instituto Nacional de Rehabilitación - INR - México.

${ }^{3}$ Médico Adscrito del Servicio de Cirugía de Columna Vertebral del Instituto Nacional de Rehabilitación - INR - México.

${ }^{4}$ Jefe del Servicio de Cirugía de Columna Vertebral del Instituto Nacional de Rehabilitación - INR - México. 
La prótesis presentó movilidad en rotación con respecto a colocación inicial en 12 pacientes. En dos casos, el implante migró con necesidad de retiro. La evaluación de EVA y EO fue de 0.7 puntos y $1.4 \%$. La ganancia de altura del espacio intersomático mejoró $2.5 \mathrm{~mm}$. Una migración a los 23 meses por esfuerzo fisico no indicado representando un $5 \%$ y no existiendo ningún hundimiento. Conclusión: se puede concluir que había una reducción del dolor y el grado de discapacidad asociada a las plataformas de menor edema y aumento del espacio intervertebral.

\section{DESCRIPTORES: Desplazamiento} del disco intervertebral/ diagnóstico; Desplazamiento del disco intervertebral/ radiografía; Biomecánica; Prótesis articulares

de $2 \mathrm{~mm}$. A prótese apresentou mobilidade em rotação durante a colocação inicial em 12 pacientes. Em dois casos o implante migrou com necessidade de remoção. A avaliação de EVA e EO foi de 0,7 pontos e 1,4\%. A ganância de altura do espaço intersomático melhorou 2,5 $\mathrm{mm}$. Uma migração aos 23 meses por esforço físico não-indicado representou $5 \%$. Não houve nenhum afundamento. Conclusão: pode-se concluir que houve uma redução da dor e do grau de incapacidade associados com menos edema das plataformas e aumento do espaço intervertebral.

\section{DESCRITORES: Deslocamento} do disco intervertebral/ diagnóstico; Deslocamento do disco intervertebral/ radiografia; Biomecânica; Prótese articular of $2 \mathrm{~mm}$. Prosthesis showed mobility in rotation during the initial placement in 12 patients. In 2 cases the implant migrated and it was necessary to remove it. The VAS evaluation and OS were 0.7 points and $1.4 \%$. The height greed of intersomatic space improved in $2.5 \mathrm{~mm}$. A migration in 23 months by non-indicated physical exertion represented $5 \%$. There was no sinking. Conclusion: We can be concluded that there was a reduction of pain and degree of disability associated with less edema platforms and increase the intervertebral space.

KEYWORDS: Intervertebral disk displacement/diagnosis; Intervertebral disk displacement/radiography; Biomechanics; Joint prosthesis

\section{INTRODUCCIÓN}

El $85 \%$ de los adultos que presentan lumbalgia mejoran sin tratamiento, pero el resto continúa con dolor crónico y disfunción, por la degeneración discal progresiva. Para estos pacientes, en la actualidad, se emplean varios procedimientos, incluyendo medicamentos y terapia física, así como la discectomía, nucleotomía, sustitución del núcleo, la artroplastía y la fusión vertebral ${ }^{1-4}$.

El disco intervertebral es una estructura compleja conformada por una pieza externa, el anulus y, una interna, el núcleo. El anulus se constituye de 12 a 20 capas de fibras de colágena tipo I en el 95\% y tiene forma de neumático sellado a alta presión ${ }^{1,5,6}$. El núcleo pulposo posee un polisacárido denominado glucosaminoglicano en un $95 \%$, cuya base principal es el AC hialurónico, que es el material más hidrotópico de la naturaleza. Lo que permite que actúe como aire presurizado en el anulus, como un neumático de automóvil. Al hincharse, el núcleo distiende al anulus apropiadamente permitiendo la movilidad controlada del segmento vertebral. El núcleo correctamente presurizado es la llave de la integridad del disco y la unidad funcional, la presión intradiscal nunca es cero incluso en cadáver ${ }^{1,4,6,7}$.

En el día, el disco está sometido a presiones relacionadas con la gravedad, lo que hace que el disco pierda volumen de hasta un $20 \%$, haciendo que al momento de acostarnos seamos más cortos, la bomba intrínseca del disco se activa en las horas de oscuridad y al estar acostado en posición horizontal, permite la regeneración y/o reparación de tejidos finos permitiendo el paso de alimentos y egresando los residuos. La función de síntesis del núcleo pulposo declina a partir de la segunda a la cuarta década de la vida, por eso es que en esta etapa de la vida, el padecimiento es más frecuente ${ }^{7-11}$.

Cuando se intenta crear una substitución de disco, las características del dispositivo deben ser semejantes al disco natural para preservar la biomecánica de movimiento y proporcionar estabilidad a la columna ${ }^{12,13}$.

Los intentos de crear discos artificiales no son nuevos, desde 1955, David Cleveland inyectó un acrílico de metilo en el espacio intervertebral, después de una discectomía, y la historia es grande hasta nuestros días ${ }^{13-24}$ (Tabela 1).

La prótesis PDN diseñada por Charles Ray y Schronmayr (Ray Médica en Minneapolis Minesota) ha sido utilizada por más de diez años a partir de 1996, y la cual está constituida por una base de un hidrogel especial envuelto por una malla de polietileno, que permite mantener la forma del implante y la absorción de líquido del mismo para elevar la altura del disco, simulando la flexibilidad o permitiendo la movilidad normal de movimiento $^{2,3,25,26}$. De 1996 al 2002 se implantaron a 423 pacientes con sólo un $10 \%$ de retiro e índice de satisfacción de un $90 \%{ }^{27}$. Sin embargo, lo restringido de las indicaciones con necesidad de que el espacio sea suficientemente grande para la colocación de los dos implantes, obligó a la creación de un implante único llamado PDN-SOLO. En la que el empaque mantenga la forma y tamaño de implante con una máxima hidratación, comprobando la posibilidad de mantener la altura del espacio intersomático, sin alterar las 
plataformas y, en caso necesario, el uso de la técnica de abordaje trans-psóas, propuesta por Bertagnoli ${ }^{28-30}$.

En los reportes publicados con experiencia de PDNSOLO, con seguimiento a seis meses, se reportan buenos resultados desde el punto de vista clínico, funcional y en el incremento y mantenimiento de la altura del disco, pero con un índice de hundimiento de un $19.6 \%$, y de extrusiones de un $8.7 \%{ }^{29,30}$. Shim et al..$^{29}$ observaron en el $82.8 \%$, incremento en los signos Modic de las plataformas con respecto a los preoperatorios. Explicando esto por la colocación de las prótesis en forma muy apretada para evitar las migraciones, aumentando la inflamación de los cuerpos vertebrales y la posibilidad de hundimiento en forma ostensible.

Por tal motivo, nosotros diseñamos una técnica de fijación de la prótesis a las plataformas en la que usando una ancla sujetamos la prótesis que al quedar menos apretada no incrementa o lesiona por presión las plataformas.

En este trabajo se reporta nuestra experiencia con seguimiento mínimo de tres años.

\section{MÉTODOS}

Se ha diseñado un estudio prospectivo y longitudinal entre todos los enfermos de hernia discal lumbar, seleccionados entre los pacientes de la consulta externa del Instituto Nacional de Rehabilitación, entre junio del 2002 y junio del 2003.

Se incluyeron a pacientes mayores de 18 y menores de 55 años, con hernia discal sintomática L2 a S1 en uno o dos niveles, que no respondieron a tratamiento médico y de rehabilitación por un lapso de seis meses, con lumbalgia con o sin datos de radiculopatía, cuyo origen se encontró en el disco intervertebral, con altura de disco de más de $5 \mathrm{~mm}$ e integridad relativa del anillo discal. Con estudios de imagen completos (placas simples y dinámicas, mielografía lumbosacra, resonancia magnética y electrofisiología con EMG y VCN) para externo.

Todos los enfermos comprendieron perfectamente el tipo de cirugía al que se les iba a someter, además de conocer las características de la prótesis con estudios acerca de su seguridad biológica, aceptando la cirugía, así como los riesgos inherentes.

Los pacientes fueron evaluados en el preoperatorio y a los tres años de seguimiento. Con evaluación de escala funcional de Oswestry y visual análoga para el dolor, con radiografías simples y dinámicas para evaluar sitio y colocación de la prótesis y con RM para valorar signos de MODIC y datos de hundimiento. La movilidad de la prótesis y el funcionamiento del ancla fueron valorados en base a la posición encontrada en los estudios de imagen del postoperatorio inmediato, determinando en base a los marcadores de la prótesis si existió, o no, desplazamiento.

A todas las variables se les aplicó análisis estadístico con teste $t$ de Student.

La prótesis utilizada es la PDN-SOLO de la compañía Ray Médica, mientras que el ancla es de la compañía Smith \& Nephew, de $3.5 \mathrm{~mm}$ de diámetro, con 3.0 de longitud, y con dos suturas sujetas al tornillo y libres con un calibre de 1 .

\section{TÉCNICA QUIRÚRGICA}

La técnica quirúrgica conforma el siguiente:

- Se coloca el paciente en decúbito dorsal en posición para disectomía y se efectúa un procedimiento clásico para el retiro de la hernia de disco, cuidando en incidir de manera mínima el anillo fibroso;

- Hay exploración del espacio intersomático para verificar la extracción completa del núcleo pulposo, así como la ubicación con respecto a la porción anterior del disco intervertebral;

- Medición de manera gradual y progresiva del espacio intervertebral mediante probadores diseñados con dicho fin de 5,7 y $9 \mathrm{~mm}$ para colocar una prótesis más adecuada.

- Se coloca el ancla mediante fluoroscopia colocándola a $1 \mathrm{~cm}$ de la porción anterior de la vértebra, con penetración de $5 \mathrm{~mm}$, en la plataforma (Figura 1);

- Se une el implante al ancla mediante sutura absorbible;

- Se introduce (Resbaladilla) para permitir la introducción de la prótesis, así como redirigirla y colocarla de manera adecuada;

- Se toma control radiológico y se anudan las suturas para fijar la prótesis el ancla (Figura 1);

- Se efectúa la reparación del ligamento posterior y se sutura por planos dejando el drenaje.

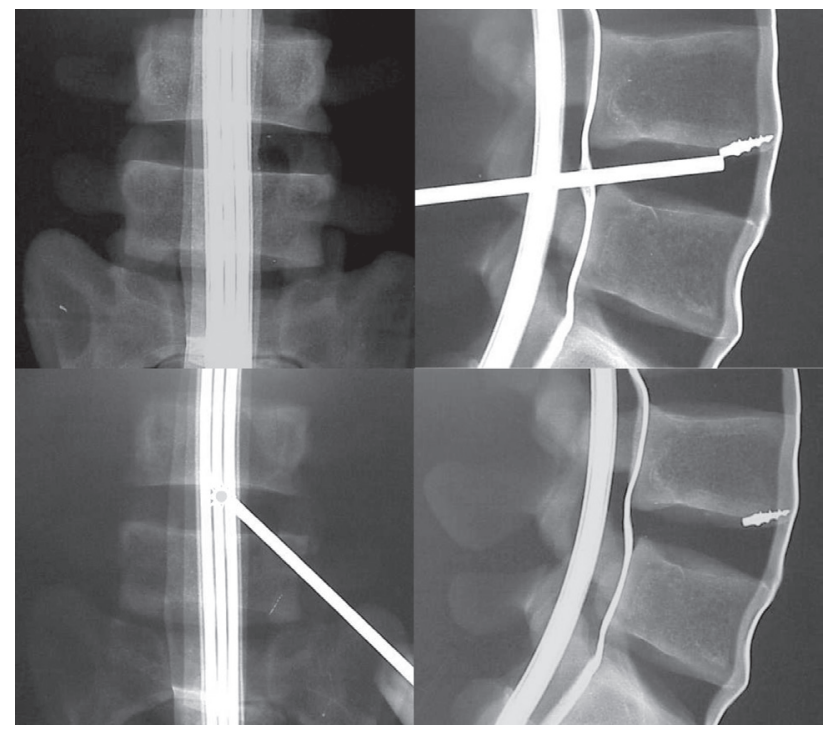

Figura 1 - Se aprecia la técnica fluoroscópica de la colocación del ancla, introduciendo la misma al cuerpo vertebral mediante un perforador y posteriormente la forma en que queda fijada. De la fijación derivan dos riendas con las que se anuda directamente la prótesis al cuerpo vertebral superior.

\section{CUIDADOS POSTOPERATORIOS}

Los cuidados postoperatorios deben ser: reposo relativo con deambulación asistida al segundo día y libre al tercero; alta en cuanto a la condición clínica y de la herida lo permita; faja lumbosacra por un período de seis semanas; y evitar durante un mes movimientos de flexión y extensión y cambios de postura en forma armónica. 
TABLA 1 - Intentos fallidos para el diseño de una prótesis de disco

\begin{tabular}{|c|c|c|c|}
\hline \multicolumn{2}{|c|}{$\begin{array}{l}\text { Prótesis basadas en la restauración } \\
\text { viscoelástica del disco. }\end{array}$} & \multicolumn{2}{|c|}{$\begin{array}{c}\text { Prótesis basadas en la restauración de la función } \\
\text { mecánica del disco. }\end{array}$} \\
\hline Diseño & Concepto & Diseño & Concepto \\
\hline Nachemson, 1950 & Inyección de silicón al disco. & Weber, 1978 & Cajas de polietileno articuladas. \\
\hline Stubstad, 1973 & Implante espiral con memoria. & Kéller, 1989 & Bases bicóncavas metálicas. \\
\hline $\begin{array}{l}\text { Hoffman-Daimler, } \\
1974\end{array}$ & Bases metálicas con espaciador plástico. & Bullivant, 1992 & Bases metálicas con centro de silicón. \\
\hline Roy-Camille, 1978 & Disco de silicón prefabricado. & Graham, 1992 & Esfera y socket dentro de bases cilíndricas. \\
\hline Edeland, 1981 & Diafragma con material higroscópico. & Salib, 1992 & Esfera excéntrica y socket. \\
\hline Downey, 1987 & Bases de polietileno con centro liquido. & Xavier, 1998 & Esfera articulada en anillo elástico. \\
\hline Ojima, 1989 & Placas de hidroxiapatita con centro de silicón. & Cauthen, 2001 & Implantes cilíndricos de encaje reciproco. \\
\hline Schoppe, 1990 & Implante inflable. & & \\
\hline Lee, 1990 & Espaciador intervertebral biocompatible. & \multicolumn{2}{|r|}{ Prótesis cervicales } \\
\hline Bao-Highham, 1991 & Hidrogel cubierto de membrana semipermeable. & Diseño & Concepto \\
\hline $\begin{array}{l}\text { Baumgartner, } 1991 \\
\text { y } 1994\end{array}$ & $\begin{array}{l}\text { Nucleoplastía espiral; Esferas elásticas } \\
\text { intradiscales. }\end{array}$ & Weber, 1979 & $\begin{array}{l}\text { Centro ovoide móvil dentro de placas } \\
\text { cóncavas. }\end{array}$ \\
\hline Beer, 1995 & Placas metálicas con resortes interiores. & Patil, 1980 & Bases metálicas con resortes internos. \\
\hline Bao, 1996 & Bastones de hidrogel expandidle. & Kehr, 1996 & Prótesis de copa intervertebral. \\
\hline Buttermann, 1996 & Pistones y resortes. & Medizadeh, 2001 & Semicilindros con resortes. \\
\hline Monteiro, 1997 & Cápsulas inflables. & Buhler, 2001 & Dos bases con centro de cerámica. \\
\hline Krapiva, 1997 & Núcleos cilíndricos expandibles in situ. & & \\
\hline Graf, 1999 & $\begin{array}{l}\text { Componente intradiscal y fijación elástica } \\
\text { transpedicular. }\end{array}$ & \multicolumn{2}{|r|}{$\begin{array}{l}\text { Elementos protésicos de } \\
\text { elementos distintos al disco }\end{array}$} \\
\hline Wardlaw, 1999 & Material transudativo e hidrogel. & Stone, 1990 & Matriz inductora de condorcitos. \\
\hline Bryan, 2000 & Placas intervertebrales articuladas. & García, 1990 & Materiales polimerizantes inyectados in situ. \\
\hline Gauchet, 2000 & Placas con núcleo deformable. & Fitz, 1996 & Prótesis facetarias. \\
\hline Jackowsky, 2000 & Prótesis elastomérica. & Martin, 2000 & $\begin{array}{l}\text { Prótesis facetarias con tornillos } \\
\text { transpediculares. }\end{array}$ \\
\hline Weber, 2001 & Prótesis de núcleo y anillo fibroso. & $\begin{array}{l}\text { Chin Chin Gan, } \\
2001\end{array}$ & Implante biodegradable intervertebral. \\
\hline Kotani, 2002 & Amillos de polietileno. & & \\
\hline
\end{tabular}

\section{RESULTADOS}

El estudio incluye 20 pacientes con un seguimiento mínimo de 36 meses y un máximo de 48 meses. El grupo comprende 8 mujeres y 12 hombres con una edad promedio de 37.6 años e intervalo de 24 a 51 años.

El tiempo promedio de la sintomatología fue de 20.9 meses desde el inicio de la primera manifestación al tiempo en que se realizó la cirugía.

Todos los pacientes fueron manejados en forma inicial con rehabilitación, baja de peso y analgésicos, persistiendo la sintomatología y siendo candidatos a cirugía.

El diagnóstico fue hernia discal extruida en los niveles de L4-L5 en 15 casos, Y L5-S1 en 5 casos. El tiempo quirúrgico fue en promedio de 142.50 minutos, con un máximo de 270 y un mínimo de 45 minutos. La estancia hospitalaria posquirúrgica fue de 1.4 días. El sangrado promedio fue de $222.5 \mathrm{~mm}^{3}$ con un máximo de 600 y un mínimo de $100 \mathrm{~mm}^{3}$.

En la escala visual análoga del dolor (EVA), la puntuación promedio inicial fue de 7.6 y a los tres años de $0.7(0.66)(\mathrm{p}<0.000)$. El 75\% (15 pacientes) requerían de analgésicos en forma diaria para mitigar el dolor.

El promedio inicial en la escala de Oswestry fue de $67 \%$. En el postoperatorio inmediato, se presentaron $32.6 \%$ y a dos años de $1.4 \%$ (de 1.85$)(\mathrm{p}<0.000)$.

El espacio intervertebral, tomando como parámetro la porción media de la vértebra en la proyección lateral, fue en promedio $7.67 \mathrm{~mm}$ antes de la cirugía, y de $10.19 \mathrm{~mm}$ a los 3 años, con una mejoría de $2.52 \mathrm{~mm}$ $(\mathrm{p}<0.000)$.

Se determinó que hubo movilización dentro del espacio intervertebral en 12 de los pacientes $(60 \%)$.

En todos los controles de IRM no se observó hundimiento de la prótesis, a pesar de verificar la movilización de la misma en 12 casos. Asimismo, no existieron signos Modic en la resonancia de control a tres años (Figuras 2 y 3). En un paciente masculino se retiró la prótesis por causar sintomatología radicular dolorosa a los 23 meses, por extrusión de la misma, provocada por esfuerzo físico inadecuado del paciente, al jugar fútbol americano, lo que representó un 5\%. 


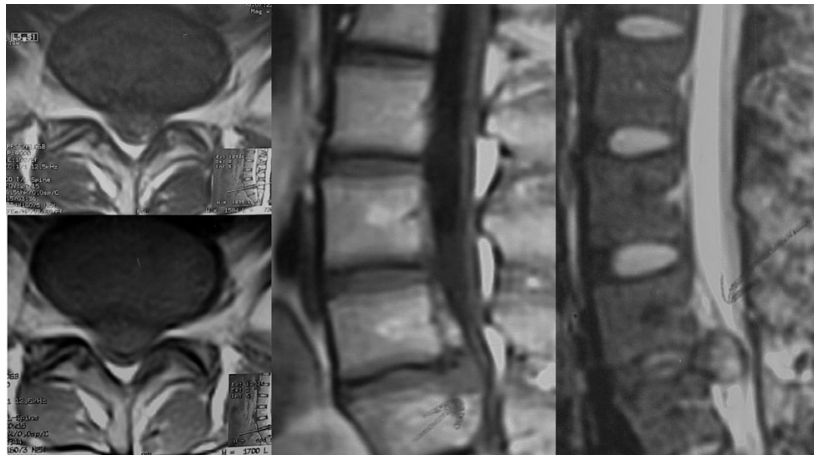

Figura 2 - Caso No. 11 - Se presenta paciente del sexo femenino de 45 años con nueve meses de evolución con dolor lumbar y radicular severo irradiado a la pierna derecha. La EVA fue de seis puntos inicial y la EO de 64\% indicando dolor y una discapacidad severa. En el estudio de RMN se aprecia una gran protrusión discal en L4-L5 que ocupa el $60 \%$ del conducto con compresión severa en la raíz de lado derecho. El manejo médico fue ineficaz, por lo que se indicó la cirugía.

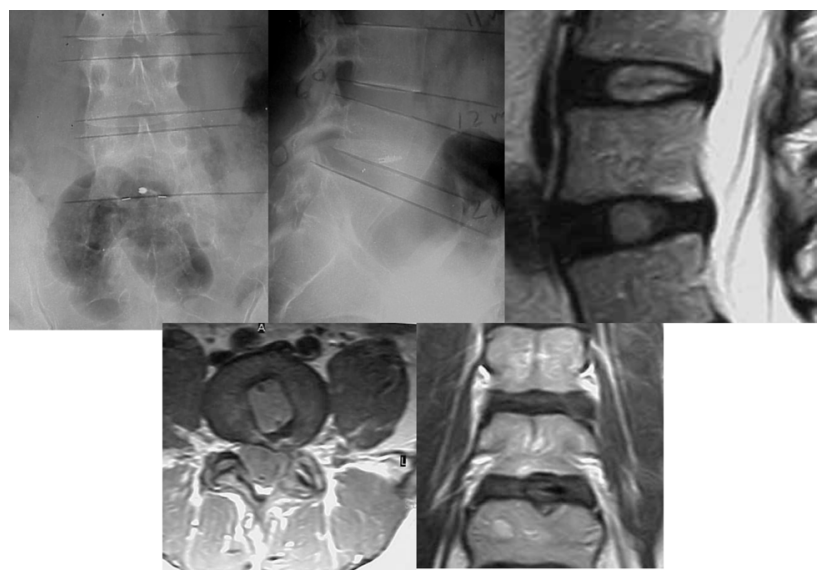

Figura 3 - Se muestra el control radiológico de la paciente con adecuada ubicación de los señaladotes de la prótesis que se aprecian como un solo punto en la placa lateral y como dos en la AP. El ancla se aprecia en posición central, justo de la espinosa. En la RM de control se aprecia también la adecuada colocación de la prótesis que no invade en ningún momento el conducto raquídeo. Al comparar las dos imágenes, se aprecia el movimiento de la prótesis dentro del espacio, sin repercusión clínica.

\section{DISCUSIÓN}

En este estudio se confirmó que la población estudiada en nuestro Instituto es, en general, conformada por adultos jóvenes y adultos con una frecuencia elevada, entre los $30 \mathrm{y}$ 49 años, en etapa económicamente activa, en donde se ven expuestos a factores de riesgo, así como la coexistencia de la degeneración discal en progreso que es acelerada por factores genéticos y ambientales a partir de las dos décadas de la vida. Con relación similar en la literatura mundial ${ }^{1,2,3,8,11-13}$.

Los pacientes del sexo masculino fueron más afectados, posiblemente a consecuencia en nuestro medio a la exposición a microtraumas repetidos sobre la región lumbar por condiciones laborales. La relación que guardó en nuestro estudio fue de 1.5:1, no conociendo con otros estudios internacionales en los cuales la relación es de 3:1. Esto tal vez sea por el número de pacientes sometidos a estudio y que posiblemente en una serie mayor sea semejante a los reportados al nivel mundial ${ }^{5-7}$.

La mayoría de los pacientes evolucionan a partir del primer síntoma durante un año hasta cinco antes del diagnóstico, debido a que llevan múltiples tratamientos conservadores encaminados a evitar la cirugía o bien, fallas en el diagnóstico antes de llegar a nuestro Instituto. Aunque el diagnóstico se puede realizar hasta en un $70 \%$, a partir del primer síntoma dentro de los seis primeros meses, lo cual también coincide con la literatura ${ }^{8,10}$.

En el presente estudio, la similitud que guardó la relación del tiempo quirúrgico y el sangrado quirúrgico fue muy similar a lo descrito, aún que con tendencias de reducción del mismo, una vez pasada la curva de aprendizaje. Siendo aún menor comparados con procedimientos considerados de mayor invasión como lo considera el doctor Bertagnoli ${ }^{2,3,8,9}$.

Uno de los objetivos del implante PDN-SOLO es de absorción de agua permitiendo el incremento de la prótesis, lo cual en teoría trata de mantener el espacio intersomático o incluso el incremento de la altura, evitando una degeneración en segmentos adyacentes. Sin embargo, en los reportes anteriores ${ }^{29,30}$ se ha observado un factor de inflamación de las plataformas, incluso el hundimiento de la prótesis por demasiada presión en las mismas, al ajustar la prótesis al tamaño para evitar su migración.

Con el uso del ancla no requerimos de usar una prótesis justa, permitiendo el incremento del dispositivo mediante su hidratación sin lesionar las plataformas por hiperpresión sobre las mismas. Una vez hidratada, comienza a funcionar como un verdadero núcleo, ya que se permite que el implante esté más relajado y funcione en condiciones más propicias con reducción del estrés de cargas del mismo, por lo que nuestros implantes aún tienen la posibilidad de mejor expansión del espacio intervertebral y esto secundariamente se vea reflejado en la reducción del choque interfacetario e interespinoso, por la estabilidad ligamentar posterior ${ }^{1,3,4,14-17}$.

Todo esto comprobado por las imágenes de resonancia magnética que demuestran la ausencia de cambios Modic, ni hundimientos en las plataformas de ningún paciente.

Tanto en la escala visual análoga del dolor como en la escala de Oswestry, apreciamos que la reducción del dolor y el grado de discapacidad preoperatorio fue satisfactorio comparado a tres años, esto refleja que se está muy por debajo del $20 \%$ que se comenta en los estudios a nivel mundial, se reserva a buena evolución posterior de una cirugía lumbar. Lo que prueba que el mismo implante colocado con la fijación que proponemos tenga mejores resultados que los reportados por los doctores doctor Shim y Choi, en la República de China y Corea, respectivamente y en cuya serie de estudios ha sido aún más corta con EVA de 6 y 12 meses superiores a tres puntos y Oswestry superiores al $15 \%{ }^{29,30}$.

En los pacientes en forma aguda tras el esfuerzo realizado a los 23 meses se retiró la prótesis tratando de evitar 
reducción de la sintomatología y mayor daño neurológico. No existió pérdida del espacio intervertebral conservando la misma altura, las escalas en la actualidad se encuentran en EVA de 1,0 punto y EO de 2\% a tres años. La migración presentada solamente fue un $5 \%$ en un periodo atribuido a un mal cuidado del paciente, con sobrepeso y esfuerzo físico inadecuado como la causa. Realizando la correlación en las publicaciones llega hasta un $8.7 \%$ y éstas en el periodo menor de tres meses, sin saber si en el seguimiento posterior se observaran más.
En conclusión, se puede mencionar que la reducción del dolor y el grado de discapacidad evaluado por EVA de 7.6 puntos y EO de $67 \%$ redujeron a 0.7 puntos y $1.4 \%$, respectivamente, a dos años y se relacionó con menor edema de las plataformas, aumento del espacio intersomático evaluado por estudios radiológicos y RMN de un $75 \%$, proporcionado por la fijación de la prótesis que a su vez redujo la migración y evitó la subsidencia en todos los casos. Por eso, proponemos el uso de esta técnica en otros centros, ya que los resultados a largo plazo tienen un índice de satisfacción de casi un 95\%.

\section{REFERENCIAS}

1. Urban JPG, Roberts S. Degeneration of the intervertebral disc. Arthritis Res Ther. 2003;5(3):120-30.

2. Ray Ch. Prosthetic Disc Nuecleus Implants. Euro Spine J. 2002;11(Suppl 2):137-42.

3. Adams MA, Freeman BJ, Morrison HP, Nelson IW, Dolan P. Mechanical initiation of intervertebral disc generation. Spine (Phila Pa 1976). 2000;25(13):1625-36.

4. Umehara S, Tadano S, Abumi K, Katagiri K, Kaneda K, Ukai T. Effects of degeneration on the elastic modulus distribution in the lumbar intervertebral disc. Spine (Phila Pa 1976). 1996;21(7):811-9.

5. Taylor TK, Melrose J, Burkhardt D, Ghosh P, Claes LE, Kettler A, et al. Spinal biomechanics and aging are major determinants of the proteoglycan metabolism of intervertebral disc cells. Spine (Phila $\mathrm{Pa}$ 1976). 2000;25(23):3014-20.

6. Guilak F, Ting-Beall HP, Baer AE, Trickey WR, Erickson GR, Setton LA. Viscoelastic properties of intervertebral disc cells. Indication of two biomechanically distinct cell populations. Spine (Phila Pa 1976). 1999;24(23):2475-83.

7. Miyamoto H, Doita M. Effects of cyclic mechanical stress on the production of inflammatory agents by nucleus pulposus and anulus fibrosus derived cells in vitro. Spine (Phila $\mathrm{Pa}$ 1976). 2006;31(1):4-9.

8. Fennell AJ, Jones AP, Hukins DW. Migration of the nucleus pulposus within the intervertebral disc during flexion and extension of the spine. Spine (Phila Pa 1976). 1996;21(23):2753-7.

9. Adams MA, Dolan P. Could sudden increases in physical activity cause degeneration of intervertebral discs? Lancet. 1997;350(9079):734-5.
10.Like M, Solovieva S, Lamminen A, Luoma K, Leino-Arjas P, Luukkonen $\mathrm{R}$, et al. Disc degeneration of the lumbar spine in relation to overweight. Int J Obes (Lond). 2005;29(8):903-8.

11.Luoma K, Riihimaki H, Luukkonen R, Raininko R, Viikari-Juntura

E, Lamminen A. Low back pain in relation to lumbar disc degeneration. Spine (Phila Pa 1976). 2000;25(24):487-92.

12.Boos N, Weissbach S, Rohrbach H, Weiler C, Spratt KF, Nerlich AG. Classification of age-related changes in lumbar intervertebral discs: 2002 Volvo Award in basic science. Spine (Phila Pa 1976). 2002;27(23):2631-44.

13. Gaetani P, Aimar E, Panella L, Debernardi A, Tancioni F, Rodriguez y Baena R. Surgery for herniated lumbar disc disease: factors influencing outcome measures. An analysis of 403 cases. Funct Neurol. 2004;19(1):43-9.

14.Lemaire JP, Skalli W, Lavaste F, Templier A, Mendes F, Diop A, et al. Intervertebral disc prosthesis. Results and prospects for the year 2000. Clin Orthop Relat Res. 1997;(337):64-76.

15.Errico TJ. Why a mechanical disc? Spine J. 2004;4(6 Suppl):151S-157S.

16.Bao QB, Yuan HA. New technologies in spine: nucleus replacement. Spine (Phila Pa 1976). 2002;27(11):1245-7.

17.Studer A. Nucleus prosthesis: a new concept. Eur Spine J. 2002;11 Suppl 2:154-6.

18.Huang RC, Girardi FP, Cammisa FP Jr, Wright TM. The implications of constraint in lumbar total disc replacement. J Spinal Disord Tech. 2003;16(4):412-7.

19.Klara. Artificial nucleus replacement: clinical experience. Spine (Phila $\mathrm{Pa}$ 1976). 2002;27(12):1374-7.
20.Korge A, Nydegger T, Polard JL, Mayer HM, Husson JL. A spiral implant as nucleus prosthesis in the lumbar spine. Eur Spine J. 2002;11(Suppl 2):S149-53.

21.Husson JL, Korge A, Polard JL, Nydegger T, Kneubühler S, Mayer HM. A memory coiling spiral as nucleus pulposus prosthesis: concept, specifications, bench testing, and first clinical results. J Spinal Disord Tech. 2003;16(4):405-11.

22.Goins ML, Wimberley DW, Yuan PS, Fitzhenry LN, Vaccaro AR. Nucleus pulposus replacement: an emerging technology. Spine J. 2005;5(6 Suppl):317S-324S.

23.Di Martino A, Vaccaro AR, Lee JY, Denaro V, Lim MR. Nucleus pulposus replacement: basic science and indications for clinical use. Spine. 2005;30(16 Suppl):S16-22.

24.Kim Y. Prediction o peripheral tears in the anulus o the intervertebral disc. Spine. 2000;25(14):1771-4.

25.Bertagnoli R, Sabatino CT, Edwards JT, Gontarz GA, Prewett A, Parsons JR. Mechanical testing of a novel hydrogel nucleus replacement. Spine J. 2005;5(6):672-81.

26. Wilke HJ, Kavanagh S, Neller $S$, Haid C, Claes LE.. Effect of a prosthetic disc nucleus on the mobility and disc height of the L4-L5 intervertebral disc postnucleotomy J Neurosurg. 2001;95(2 Suppl):208-14.

27.Bertagnoli R, Karg A, Voigt S. Lumbar partial disc replacement. Orthop Clin North Am. 2005;36(3):341-7.

28.Anaya S., Vergara H. Prótesis discal de núcleo por vía anterior, tratamiento opcional en disrupción interna de disco. Reporte de un caso. Acta Ortop Mex. 2005;19(6):273-9. 
29. Shim CS, Lee SH, Park CW, Choi WC, Choi G, Choi WG, et al. Partial disc replacement with the PDN prosthetic disc nucleus device: early clinical results. J Spinal Disord Tech. 2003;16(4):324-30.
30.Jin D, Qu D, Zhao L, Chen J, Jiang J. Prosthetic disc nucleus (PDN) replacement for lumbar disc herniation: preliminary report with six months' follow-up. J Spinal Disord Tech. 2003;16(4):331-7.

\section{Correspondencia:}

Alejandro Reyes-Sánchez

Camino a Santa Teresa, 1055 - 950

Col. Héroes de Padierna

Magdalena Contreras

México - 10700, D.F.

E-mails: alereyes@inr.gob.mx; areyes@ vertebrae.com.mx 